\title{
Tratamiento de la constipación crónica del lactante con avena machacada y harina de avena
}

\author{
Nelly Pak D. '; Eduardo Atalah S.'; Patricia Bustos M.'; \\ Marcela Alviña W. ${ }^{1}$ : Elinor Zumelzu C. ${ }^{2}$
}

\section{Oat products in the management of infant's constipation}

\begin{abstract}
The effect of crushed oal and oat flour added to milk feedings was evalucted on monagement of infont's constipction. Ninery two infarils with perșistenl constipation were selecled (mean age $6.5 \pm 1.8$ month) aric civided in Ihree groups whose milk. feechings were respeclively given supplemenls of crushed oul, oar flour or no supplemenl contrals) in addition to the ususal dierary indications for constipolion treatment. The effect wos evaluated by means of c recall of mother's opinior at 7 and 14 doys ofier beginning of trealment and daily written records on number and harciness of intants slools. Increased frequency of evacuation and decreased hardness of the stools was observed with both products. These trends were stalistically sigrificcnt, porticuiarly at the second week of treatment. Tolerance and acceptance were good for bolh products. Cherical composition and energy conlent of crushed oat arid oat flour were similar ra those of other cereals used in infants feeding. Adminisiration of these products for longer time periods could probably result in improved effect.
\end{abstract}

[Key words: constipolion, cereals, col, dienl fiber, foed, formulated.'

Aunque no hay datos precisos sobre la prevalencia de la constipación en el lactante, se estima que en EE.UU. afecta a $3 \%$ de los niños que asisten a control de salud y causa $25 \%$ de sus consultas gastroenterológicas'.

Las características de las deposiciones de los lactantes varían ampliamente en frecuencia y consistencia, por lo que no existe consenso para definir la constipación. En general, se acepta que es una evacuación incompleta, difícil, que causa molestia o dolor. Sus manifestaciones clínicas más frecuentes son meteorismo, cólicos, dolor al evacuar, rectorragia, malestar general y anorexia ${ }^{2}$. La evolución de la constipación puede ser aguda o crónica, esto último cuando los síntomas duran más de tres meses y no responden a los tratamientos habituales. Según su patogenia puede ser funcional u orgánica, secundaria a amplia gama de trastornos. La mayor

1. Departamento de Nutrición, Facultad de Medicina. División Norte, Universidad de Chile.

2. Alumna del Magíster de Nutrición, Facultad de Medicina, Universidad de Chile.

Financiado parcialmente por Sociedad Molinera E] Globo, que suministro los producıos de avens. parte de los casos de constipación crónica en lactantes se debe a causas funcionales, donde los factores dietéticos pueden jugar un papel importante. Entre ellos se puede mencionar la utilización de leche de vaca, por su alto contenido de calcio y caseína, y el escaso consumo de fibra dietética ${ }^{3-5}$. El tratamiento de estos pacientes tiene como propósito conseguir y mantener el vaciamiento rectal completo. Para ello se recomienda, entre otras medidas, aumentar la ingestión de fibra, aunque las posibilidades de incorporarla en la alimentación de los lactantes son limitadas. Generalmente se sugiere, además, el consumo de jugos de frutas (cuyo contenido de fibra es muy bajo) y aumentar el aporte de veduras y frutas.

Dado su contenido de fibra, la avena se ha usado tradicionalmente en el manejo de la constipación, incorporándola a fórnulas lácteas o comidas, siendo bien aceptada por la madre y por los niños. A pesar de su empleo frecuente en el tratamiento de la constipación del lactante, no existen antecedentes bibliográficos sobre su eficacia, por lo que se realizo la siguiente evaluación clínica controlada, comparando los re- 
sultados con los de otras medidas. El estudio se complementó con el análisis de la composición química y del contendio de fibra dietética soluble e insoluble de los productos preparados.

\section{Material y Método}

Se estudiaron 92 lactantes con constipacion, de 4 a 9 meses de edad, seleccionados de cuatro consultorios del Servicio de Salud Metropolitano Norte (La Pincoya, Quinta Buin, Lucas Sierra y $\mathrm{N}^{*} 2$ ), los que fueron seguidos por un periodo de 14 días. La seleccion fue hecha por enfermera en controles de salud, definiendo constipación habitual por la existencia de deposiciones duras o escasas, asociada a dificulad cn la evacuación, por un períado continuo o intermitente superior a quince días. Se excluyeron del estudio los nifios alimentados con lactancia materna, que estuvieser recibiendo tratamiento farmacológico o sufriesen desnutrición o enfermedades gastrointestinales asociadas.

En cada consultorio se formaron de manera aleatoria tres grupos: uno de control (n: 30 \} fal que le se recomendó emplear medidas dietéticas generales consistentes en aumento de la ingestion de líquidos, verduras $y$ frutas); otro (n: 32 ) que recibio avena machacada $5 \%$ somelida a cocción por ebullición durante 10 minutos e incorporada a las mamaderas despućs de colarla, además de las medidas indicadas a los controles $y$, finalmente, uno $(0: 30)$ en que se prescribio incorporar, a los biberones, harina de avena $4 \%$ después de cocerla por ebullición durante 5 minutos y sin colarla, además de las medidas dieteticas mencionadas. La fórmula de los biberones se hizo de la misma manera en los tres grupo, con leche en polvo de $26 \%$ en materia grasa, diluida al $10 \%$, estandarizando su preparación mediante entrega a las madres o cuidadoras de medidores especiales para la leche. La avena rachacada se obtiene industria]mente por aplastamiento mecánico del grano de avena (limpio y desprovisto de tegumento) y la harina de avena es un subproducto de la elaboración industrial del salvado de dicho cereal. Durante el control de salud la enfermera enseñó a las madres a preparar en forma correcta la avena o batina de avena según cotrespondiera, entregándoles los alimentos, el material para facjlitar la preparacion (ntedidores y coladores, un iupreso con instrucciones para la preparación de la fórmula láctea y formularios para registrar diariamente las caracteristicas de las deposiciones (frecuencia y consistencia) y el volumen de leche consumido en cada mamadera durante los 14 días estudiados.

Al séptimo día de incorporado al estudio, cada lactante fue visitado en $5 u$ domicilio por una sutricionista o un mádico, que obtuvieron información sobre las características de la constipación antes de ingresar al protocolo (duracín, frecuencia y consistencia de las deposiciones) y el uso de medicamentos para su tratamiento. Además tealizaron una encuesta alimentaria por técrica de recordatorio del día anterior y revistron los formularios de registro de las caracteristicas de las deposiciones y la aceptabilidad de las fómulas lácteas. En caso necesano, se reforzaron las indicaciones de preparación de las manaderas y el modo de aplicar los formularios. A los catorce días se repitió la visita domicilliaria pura evaluar el cumplimiento de las indicaciones de la primera visita y retirar los formularios.
E] valor nutritivo y el contenido de fibra dietética (total $y$ fracciones soluble $e$ insoluble) en avena machacada y harina, se realizó paralelamente en las preparaciones hechas de acuerdo a las indicaciones impartidas. Se determino bumedad, cenizas, lípidos, proteínas ( $\mathrm{N} x$ 6,25) y fibra cruda, según los métodos de la AOAC ${ }^{6}$. Los extractivos no nitrogenados se estimaron por diferencia y el aporte energético se calculo aplicando los factores de Atwater. La fibra dietélica totad, soluble e insoluble, se determinó en triplicado?

En el analisis estadístico se utilizó la prueba de chi cuadrado y el análisis de varianza, considerándose como siguificativas diferencias con $p<0,05^{8}$.

\section{Resultados}

La edad de los niños estudiados fue $6.6 \pm 1,8$ meses, con márgenes de 4 y 9 meses y de distribución muy parecida entre los tres grupos (p: ns). En $46 \%$ de los niños se registraba constipación desde el nacimiento y en $33 \%$ la duración de ésta era igual o mayor a tres meses (tabla 1). Cerca de la mitad de los lactantes tenía evacuaciones diariamente, el resto con menor frecuencia, en algunos casos extremos sólo dos veces por semana (tabla 2). Todos los niños es-

\section{Tabla 1}

iuración de la constipación antes del tratamiento

\begin{tabular}{lcccr}
\hline $\begin{array}{l}\text { Dur. lón } \\
\text { (meses) }\end{array}$ & $\begin{array}{c}\text { Avena ma- } \\
\text { chacada (\%) }\end{array}$ & $\begin{array}{c}\text { Haring de } \\
\text { avena }(\%)\end{array}$ & Control & Total \\
\hline$\leq 1$ & 22 & 3 & 10 & 12 \\
2 & 16 & 3 & 10 & 9 \\
$\geq 3$ & 25 & 40 & 33 & 33 \\
Desde nac. & 37 & 53 & 47 & 46 \\
\hline Total & 100 & 100 & 100 & 100 \\
\hline
\end{tabular}

$\chi^{2}: 0,32 p:$ ns

Tabla 2

Frecuencia de evacuaciones antes del tratamiento

\begin{tabular}{lcccc}
\hline $\begin{array}{l}\text { Frecuencia } \\
\text { (dias) }\end{array}$ & $\begin{array}{c}\text { Avena ma- } \\
\text { chacada (\%) }\end{array}$ & $\begin{array}{c}\text { Haring de } \\
\text { avena }(\%)\end{array}$ & Control & Total \\
\hline Diaria & 41 & 57 & 43 & 47 \\
Cada dos dias & 47 & 30 & 37 & 38 \\
$Z 3$ dias & 12 & 13 & 20 & 15 \\
\hline Total & 100 & 100 & 100 & 100 \\
\hline
\end{tabular}

$\chi^{2}: 2,82$ p: ns. 
tudiados presentaban deposiciones duras -una de las condiciones para ingresar al protocolo-, que en un tercio de ellos constituían pequeñas masas duras redondas (escíbalos). La duración de la constipación, frecuencia y características de las deposicjones fueron semejantes entre los 1res grupos. Cincuenta y siele por ciento de los lactantes no había recibido tratamiento antes de ingresar, en el resto se habían tomado medidas dietéticas como eliminar el arroz y aumentar el aporte de frutas y verduras (32\%) o farmacologicas $(11 \%)$. La proporción de niños con tratamiento previo fue algo mayor en el grupo control (43 vs $27 \%$ ), sin embargo, la diferencia no fue estadísticamente significativa.

Al término de la primera semana de seguimiento se constató que $85 \%$ de las madres preparaba la leche en una concentración adecuada, proporción que aumentó a $94 \%$ en la segunda semana del estudio. En los grupos experimentales todas usaron el espesante indicado. En el grupo control, $70 \%$ empleó algún espesante (que no era avena) y las demás ninguno. La mayoría (91\%) de los niños recibía líquidos adicionales a las comidas $(151 \pm 104 \mathrm{ml}$ por día), especialmente agua ( $47 \%)$, jugos de fruta $(16 \%)$ o ambos $(28 \%)$. El consumo de jugos era mayor en el grupo control $(p<0,02)$. La encuesta alimentaria realizada en la segunda semana del estudio mostró una situación similar.

La aceptación de las fórmulas lácteas experimentales por parte de los niños fue buena, por lo general, en opinión de las madres, pues $79 \%$ de ellos acept $\delta$ bien la mamadera con avena (machacada o como harina), a 3\% no le gustó y en $18 \%$ fue regular, mejorando en $64 \%$ de estos últimos entre la primera y segunda semana de observación. El volumen de leche consumido cn cada marnadera durante los 14 días de observación, obtenido por registro diario, permitió tener información en alrededor de 21 mamaderas por semana y por nin̄o. Durante la segunda semana, $67 \%$ de las mamaderas ofrecidas fueron consumidas en su totalidad y en otro $22 \%$ adicional el consumo fue superior a $50 \%$, lo que refleja una adecuada aceptación. La preparación de las mamaderas con los productos de avena no ofreció dificultades a las madres $(87 \%)$, aunque $20 \%$ de las que debieron emplear la harina expresaron haber tenido alguna dificultad en diluit el producto (p: ns).

Según las madres, con ambos tratamientos se obtuvo efecto positivo en $86 \%$ de los casos, pues aument 6 la frecuencia de las deposiciones, disminuyó su consistencja o se modificaron ambas. El mismo hecho se comprobó con el registro diario, con aumento del número de deposiciones, cuyo total es estadísticamente significativo desde la primera semana ( $\mathrm{p}<0,01$ ), fenómeno que se hizo más nítido durante la segunda semana de observación para ambos productos derivados de la avena. El promedio de deposiciones semanales formadas y pastosas fue mayor en los grupos con avena. Por el contrario, las deposiciones duras predominan en el grupo control. El número de deposiciones totales fue notoriamente superior en los grupos tratados con avena. La distribución porcentual según tipo de deposiciones para la primera y segunda semana de tratamiento se presenta en la tabla 3. En el grupo control predominan las deposiciones duras, tanto en la primera como en la segunda semana, mientras que en los niños que reci-

Tabla 3

Distribución según tipo de deposiciones đurante la primera y segunda semana estudiada

\begin{tabular}{|c|c|c|c|c|c|c|c|c|}
\hline \multirow[t]{2}{*}{$\begin{array}{l}\text { Deposiciones } \\
\text { tjpo }\end{array}$} & \multicolumn{2}{|c|}{ Avena (\%) } & \multicolumn{2}{|c|}{$\begin{array}{c}\text { Haring } \\
\text { de apena }(\mathscr{K})\end{array}$} & \multicolumn{2}{|c|}{$\begin{array}{c}\text { Control } \\
(\%)\end{array}$} & \multicolumn{2}{|c|}{$\begin{array}{c}\text { Total } \\
(\%)\end{array}$} \\
\hline & S1* & $S 2^{* *}$ & $\mathbf{S} 1^{*}$ & $\mathbf{S 2 * *}$ & $\mathbf{S} 1 *$ & $\mathbf{S}^{2 * * *}$ & $S 1^{*}$ & $S 2 \neq *$ \\
\hline Duras & 38 & 24 & 38 & 23 & 65 & 49 & 46 & 30 \\
\hline Formadas & 36 & 54 & 30 & 52 & 19 & 35 & 29 & 48 \\
\hline Pastosas & 26 & 22 & 32 & 25 & 16 & 16 & 25 & 22 \\
\hline Total & 100 & 100 & 100 & 100 & 100 & 100 & 100 & 100 \\
\hline
\end{tabular}

S1: primera semana 
bieron avena predominan las deposiciones de menor consistencia. Este hecho fue aún más acentuado durante la segunda semana de observación.

En la tabla 4 se describen la composición química, el aporte de energía y el contenido de fibra dietética de las preparaciones de avena machacada y harina (cocción por 10 minutos al $5 \%$ de concentración y colando 0 cocción por 5 minutos al $4 \%$ de concentración sin colar), destacando el aporte de fibra de la harina de avena, especialmente de la forma insoluble.

\section{Comentario}

La constipaciôn crónica del lactante es frecuente $y$ no siempre es tratada, a pesar de la importancia que la madre le atribuye y los síntomas que produce en el niño. La información sobre la duración de la constipación antes del estudio proviene, sin enbargo, de una entrevista efectuada a la madre y no había sido comprobada por el equipo de salud.

Debida a que los niños fueron seleccionados injcialmente al azar, las características de los grupos en relación a la duración de la constipación antes del tratamiento no resultaron idénticas. Como en otras experiencias, el Itastorno aparece precozmente e incluso, como en algunos de nuestros casos, desde el nacimiento, por lo que su tratamiento probablemente ofrece mayor dificultad". El porcentaje importante de lactantes $(57 \%)$ no tratados antes de ingresar al protocolo refleja la poca relevancia que aparentemente se otorga al problema. Las deposiciones duras persistentes pueden causar inflamación crónica -en algunos casos intensa-, fibrosis del esfínter intemo y perpetuar la constipación ${ }^{10}$. Su oportuna detección y tratamiento permitirían también cvitar los problemas sicológicos que se asocian con la afección a edades posteriores ${ }^{11}$.

El gran número de niños del grupo control que no utilizaban espesante en las formulas lacteas es inconveniente, porque recibían menor aporte calórico y les faltaba la contribución del cereal en aumentar la masa fecal y aliviar la constipacion.

Con frecuencia las madres intentan tratamientos dietéticos, que habitualmente no corrigen el problema, pues los jugos de frutas (que
Tabla 4

Composición quimica, aporte de energla y contenido de fibra dietética de las preparaciones con avena aplastada y harina de avena

\begin{tabular}{|c|c|c|}
\hline Compasicion & \multicolumn{2}{|c|}{$(\mathrm{g} / 100 \mathrm{ml})$} \\
\hline Proteínas & 0,30 & 0,47 \\
\hline Lípidos & 0,14 & 0.16 \\
\hline Energia"-. & 9,80 & 14.80 \\
\hline \multicolumn{3}{|l|}{ Fibra dietética } \\
\hline Insoluble & 0,08 & 0,20 \\
\hline Soluble & 0,07 & 0,10 \\
\hline Total & 0.15 & 0,30 \\
\hline
\end{tabular}

* al $5 \%$ y colada

$* \quad$ al $4 \%$

*** kcal $\cdot 100 \mathrm{ml}$

son colados) aportan bajísimo contenido de fibra dietética, por lo que su indicación, desde este punto de vista, no sería necesaria, a menos que la constipación obedezca a escasez relativa de agua en los alimentos por exceso de concentración de sólidos. El jugo de ciruelas tiene, sin embargo, buena acción laxante, debido a que contiene dihidroxi-fenil-isatina. La avena machacada y la harina de este cereal parecen ser una opción eficaz en el tratamiento de la constipación crónica del lactante, son bien toleradas y acepladas, además que fáciles de preparar. Los principales efectos de la harina de avena y el machacado son aumentar la frecuencia de las deposiciones y disminuir su dureza significativamente, lo que se bace más notorio con el tiempo. Ambos tipos de fibra contribuyen a aumentar el volumen de las deposiciones: la insoluble, por no ser digerible y la soluble mediante la fermentación bacteriana que ayuda a incrementar la masa microbiana del contenido fecal $^{12}$.

A pesar que los niños que recibieron harina de avena presentabar, antes del tratamiento, la constipación más acentuada, en ellos se registró la mejor respuesta. Esto puede deberse a que Ja preparación, a pesar de emplearse en concentración de $4 \%$, aportres, pero se prefirió a éstos: I.

La composición química y el aporte energético de la avena machacada y la harina de avena son comparables con los de otros cereales utilizados en la alimentación del lactante ${ }^{13}$ en simi- 
Lar dilucion. Esta información permite estimar el aporte nutritivo de la preparación de la avena tal como se consume. Aparentemente, el contenido de fibra dietética por $100 \mathrm{ml}$ de preparación es bajo, sin embargo, la ingestión tolal diaria por kilo de peso se aproxima bastante a la inferior recomendada para el adulto (no existen recomendaciones para lactantes $)^{14}$. Cabe hacer notar que el lactante también recibe fibra dietética adicional con las comidas. Entre los dos productos de avena analizados, la harina aparentemente tiene ventajas, porque son mayores el aporte energético y de fibra y es menor la manipulación requecida para prepararla. Es posible que las constipaciones que se tratan regularmente en los servicios de atención primaria sean leves o moderadas, como en los pacientes de nuestro estudio. La falta de respuesta al tratamiento de algunos niños a avena machacada o en harina pudiera deberse a casos de constipación orgánica aún no identificados, que requieren tratamiento especializado ${ }^{15}$. Los niños incluidos en estas series recibian sólo alimentación artificial láctea y sólida, porque la lactancia materna previene la constipación, dadas las características químicas de la leche humana.

Estos resultados sugieren que la avena machacada y la harina de avena pueden ser útiles en el tratamiento de la constipación crónica del lactante. Sin embargo, para lograr mayor efecto podría ser recomendable evaluar el uso de estos productos por un período más prolongado.

\section{Resumen}

Con el propósito de evaluar el efecto del uso de avena machacada y de harina de avena (subproducto de la fabricación de salvado de avena) en la constipación del lactante, se seleccionaron 92 niños con constipación habitual (edad promedio $6,6 \pm 1,8$ meses), formando un grupo que recibio avena machacada $(n=32)$, otro harina de avena $(n=30)$, ambos agregados a la leche $y$ uno de control $(\mathbf{n}=30)$. Todos los niños recibieron, además, las indicaciones dietéticas habituales (aumentar la oferta de líquidos, yerduras y frutas) en el manejo de la constipación. Los desenlaces se midieron con entrevista de opinión a la madre a los 7 y 14 días del inicio del tratamiento y registro diario del número y de las características de las deposiciones. Los resulta- dos mostraron aumento de la frecuencia y disminuciớn de la dureza de las evacuaciones con ambos productos de avena, estadísticamente significativos desde la primera semana de observación, pero más notorios durante la segunda. Los derivados de avena fueron bien tolerados y aceptados; su composición química y aporte de energía son comparables con los de otros cereales usados en la alimentación del lactante. La administración de estos productos por mayor tiempo podría mejorar el efecto observado.

(Palabras clave: constipación, cereales, avena, fibra dietética.)

\section{Referencias}

1. Clayden GS: Constipation in childhood. BMJ 1989; 299: 1116-1117.

2. Krebs $C$ : Constipación inlestinal, En Pediatria. Meneghello J, Fanta E. Paŕs E, Rosselor J. Ed. Medifertáneo, 4 Ed. Santiago, 1991: 1126-1131.

3. Floch $M$ : The pharmacology of dietary fiber for laxation. Am J Gastroenterol 1987: 82: 1295-1296.

4. Jenkins DJ, Peterson RD. Thome MJ. Ferguson PW: Wheat tiber and laxation: dose response and equilibralion time. Am J Gastroenterol 1987; 82: 1259-1263.

5. Taylor $R$ : Management of constipation. Br Med J 1990: 300: 10.634 .

6. Official Methods of Analisys. I3th Ed. AOAC, Arlington, VA, 1980

7. Asp NG, Johansson CG, Hallmer $H$, Siljestrom $M$ : Rapid enzimatic assay of insoluble and soluble dietary fiber. J Agric Food Chem 1983; 31: 476.

8. Snedecor GW, Cochran WG: Statistical Methods. Iowa. The Iowa Siate University 1972.

9. Abrahamtian FP. Lloyd-Still JD: Chronic constipation in childhood: a lingitudinal study of 186 patients. $J$ Pediatr Gastroenterol Nulr 1984; 3: 460-467.

10. Krebs C. Flores G. Bravo 1, Cruz MA, Sepulveda $R$. Rojas S: Electromanometria anorrectosigmoídea en el diagnóstico diferencial de la constipación en el nìno. Rev Chil Pediatr 1988; 59: 11-16.

11. Larraín F: Constipación: Enfoque médico. Rev Chil Pediatr 1988; 59 (supl. 2) : 39-41

12. Kritchevsky D; Dietary Fiber. Ans Rev Nutr 1988: 8: 301-328.

13. Schmidt-Hebbel H, Pennucchiont I, Masson L y Mella MA: Tabla de Composición Qưnica de los Alimentos Chilenos. Fac. Cs. Químicas y Farmacéuticas. Depto. Ciencias de los Alimentos y Tecnología Quítnica. Universidad de Chile. Santiago, Chile.

14. Center for food safety and applied nutrition food and drg administration. Physiological effect and health consequences of dietary fiber. Life Sciences Research Office. Federation of American Societies for Experimental Biology. June 1987

15. Larrafn $F$ y Danís $O$ : Constipación crónica idiopatica. Estudio longitudinal en 40 casos. Rev Chil Pediatr 1992; 63: 205-208. 